\title{
A Review on Optical Fibre Sensor Topology and Modulation Technique
}

\author{
S. Nurulain', N.N. Mazlee ${ }^{1}$, M. R. Salim², H. Manap ${ }^{1}$ \\ ${ }^{I}$ Faculty of Engineering Technology \\ University of Malaysia Pahang \\ Pahang, Malaysia. \\ ${ }^{2}$ Faculty of Electrical Engineering, \\ University of Technology, Malaysia, \\ Johor, Malaysia. \\ Email: ainnadhirah93@gmail.com
}

\begin{abstract}
This paper reviews of optical fibre sensor and it focuses on sensor topology and modulation techniques. Fundamentals and working principles of optical fibre based sensing system are thoroughly explained. Particular interest is given on various technique and highlight its advantages and disadvantages. The objective of the review is to evaluate their respective suitability for use as a sensor in different environment or it can provide an alternative method of optical sensing in any particular sector.
\end{abstract}

Keywords:

Optical fibre sensor, topology, modulation technique

\section{INTRODUCTION}

Optical fibres were becoming widespread in their commercial use with the telephone transmission systems and other communication systems. They are also widely used in medical imaging and mechanical engineering inspection but purely as light illumination light guides. The use of optical fibres in sensing application has become more widespread since they were initially investigated in the late $70 \mathrm{~s}$ and early $80 \mathrm{~s}$. There are many examples of these early developments and these have been thoroughly reviewed recently by Lyons [1].

There has been much progress in the advancement of standard optical fibres since 1970s, such as early production of $\left(20 \mathrm{dBkm}^{-1}\right)$ optical fibre, first appearing in the development of Plastic Optical Fibre (POF), and the invention of easy-to-use fusion splicer for single mode fibre, which allows more accurate alignment and reduces splice loss. Although much of the progress was stimulated by the telecommunications industry, all of these achievements have an indirect impact on the progress of the optical fibre sensing system. Development of optical fibre sensors began intensely in 1977 although there were some isolated demonstrations before this date as reviewed by Lyons [1].

Optical fibre sensors have been and are used to measure strain, temperature, pressure, flow, vibration and many other physical quantities. Based on the trend of $15^{\text {th }}$ Optical Fibre Sensor conference (OFS-15), Portland, Oregon, USA, 2002 shown in Figure 1, strain measurement was the most popular research being performed followed by temperature measurement [[2]]. In most measurements, fibres are modified so that the quantity to be measured modulates the intensity, phase, polarization, wavelength or transit time of light in the fibre. Sensors that modulate the intensity of light are the simplest, since only a simple source and a detector are required. Also optical detectors fundamentally only allow the measurement of intensity. The other techniques incorporate the measurement of light intensity in one form or another e.g. a modern spectrometer as the one used in this investigation comprises an array of intensity detectors into a CCD array. 
INTERNATIONAL JOURNAL OF ENGINEERING TECHNOLOGY AND SCIENCES (IJETS) Vol.7 (1) June 2017 DOI: http://dx.doi.org/10.15282/ijets.7.2017.1.7.1069

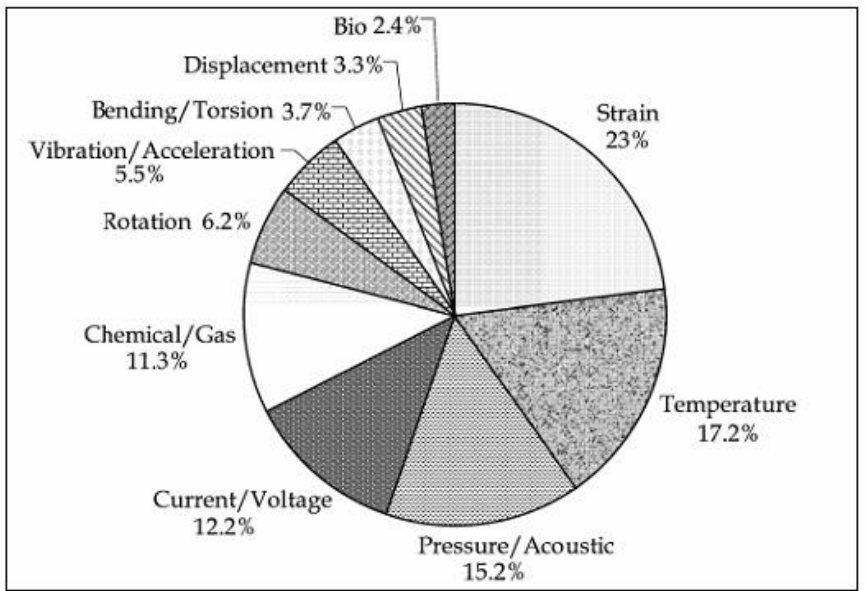

Figure 1: Distribution of OFS-15 proceeding according to measurands [2].

Since numerous optical fibre sensors have been developed to measure different types of quantities, a few reviews have been carried out on optical fibre sensing systems as early in 1982 by Giallorenzy et al [[3]] and A.L. Harmer [[4]]. In 1996, A.D. Kersey [[5]] demonstrated in his review that optical fibre sensors had already been used over a very diverse range of areas. Due to the rapid progress in this field, a review that covers all types of optical fibre sensors in a few-page article is almost impossible. In his review paper, A.D. Kersey [5] concentrates on a limited type of optical fibre sensors such as interferometric sensors, intrinsic distributed sensors and Bragg grating based systems. There are also reviews on other types of optical sensing system such as Fibre Optic Gyroscopes (FOG) by Byoungho Lee [[2]] and multiplexed sensor by Grattan et al [[6]]. In this paper the review will only focus on sensor topology and modulation technique.

\section{SENSOR TOPOLOGY}

There are several ways on how a sensor or a sensor system can be configured. Generally, it is based on the spatial distribution of the measurands and can be classified as the Single Point Sensor, Distributed Sensor and Quasidistributed Sensor

\subsection{Single Point Sensors}

Generally single point sensor measurements are carried out at a single point often located at the end of the fibre as shown in Figure 2. However, it is not necessary to measure only one point of the spatial distribution of the measurands. It is possible to install as many sensors as may be required to address multiple sensing points.

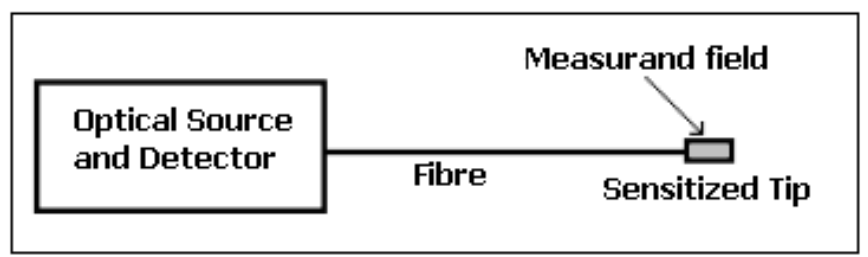

Figure 2: Single point sensor.

In practice, where there are a large number of points to be measured, it is not practical to use a multiple single point sensor. This is due to problems faced in complexity of installation of the sensing system and elevated cost. Besides having too many point sensors, many fibres are required to connect the sensor to the sources and detectors. However there is an effective solution to this problem using optical fibres. It can be solved by multiplexing all the sensing points onto a single optical fibre. Point sensors can be used in wide areas such as measuring temperature [78], gas detection [[8]] and water quality assessment [[9]]. 
INTERNATIONAL JOURNAL OF ENGINEERING TECHNOLOGY AND SCIENCES (IJETS) Vol.7 (1) June 2017 DOI: http://dx.doi.org/10.15282/ijets.7.2017.1.7.1069

\subsection{Distributed Sensors}

As discussed above, there are disadvantages of using point sensors. Thus using distributed sensors is a better option for certain measurements, for example, temperature measurement along underground power cable up to $30 \mathrm{~km}$ long by York sensor's DTS 800 [[10]]. For an optical fibre distributed sensor, the parameter of interest is measured at any point or continuously along a single optical cable with a certain spatial resolution as shown in Figure 3. It can simultaneously measure up to 10,000 points on a single optical fibre [[12]] hence will significantly reduce the cost of fibre usage and installation.

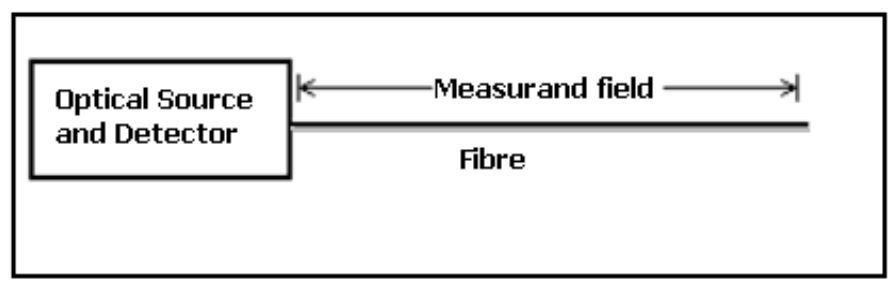

Figure 3: Basic diagram of distributed sensor.

However, optical fibre distributed sensors also have some drawbacks. Generally, distributed optical fibre sensors can only be used for static or slow varying measurements such as static strain or temperature measurement [[13]]. According to Zhang [[13]], a dynamic measurement using the distributed sensor is difficult to achieve because of the large number of waveforms generated for the signal processing and averaging. Another disadvantage of a distributed sensor is that the scattering signal of the long optical fibre is weak, resulting in low signal-to-noise ratio (SNR). Hence a more powerful light-source and more complicated electronics and detection method have to be employed. This is particularly the case with non linear methods such as Raman and Brillouin Scattering [[14]].

The principle of optical fibre distributed sensors is used in various types of measurements. Besides temperature measurement [[15]], distributed sensors can be used to measure stress and strain on a large structure [[16]], vibration monitoring on a long bridge [[17]] and many other applications such as pressure, current and chemical concentration measurement [[18]]. The above are examples of continuous spatially resolved distributed methods. There are several examples in which distributed measurement occurs but no spatial resolution is present. In these cases the signal is integrated or averaged along the length of the sensor. An example of this type is reported by Fitzpatrick et al [[19]].

\subsection{Quasi-Distributed Sensors}

Quasi-distributed sometimes known as multi-point or multiplexed sensors are another variation of distributed sensors system. In quasi distributed sensor configurations, the measurand is not monitored continuously along the fibre path, but at a finite number of locations as shown in Figure 4.

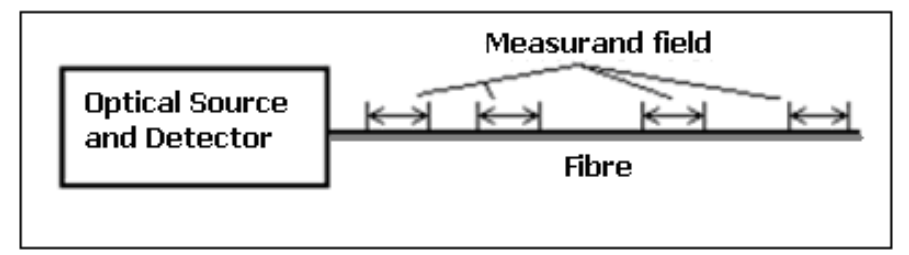

Figure 4: Distributed sensors.

Quasi distribution is accomplished by locating limited sized sensor elements at discrete locations along the fibre. For quasi-distributed sensors, fibre Bragg gratings are often used as the sensing element due to its long distance sensing capability [[20]] and its ability to measure a number of different physical characteristics simultaneously [[21]]. Quasi-distributed sensors are used in various applications and can perform single-measurand monitoring applications such as measuring $\mathrm{pH}[[22]]$. It is also possible to operate multi-measurand monitoring by sensitizing each sensor element to specific measurand field of interest [[23]]. 
INTERNATIONAL JOURNAL OF ENGINEERING TECHNOLOGY AND SCIENCES (IJETS) Vol.7 (1) June 2017 DOI: http://dx.doi.org/10.15282/ijets.7.2017.1.7.1069

\section{Sensor Modulation TeChniQueS}

In optical fibre sensor system, optical signal or light is modulated when it is propagated in the measurand field before it is sensed by the detector. There are a few techniques used to modulate the optical signal based on measurement purpose and equipment used for detection system. Usually, light modulation techniques are classified in a few segments such as intensity modulation, wavelength modulation or time modulation. There are other methods used for light modulation such as frequency modulation and phase modulation. These are just other variations of the modulation techniques and the basic operating principle is the same. For example, frequency modulation is a form of wavelength modulation since they are inversely proportional [[24]]. There are other modulation techniques available such as polarisation modulation [[25]] and refractive index modulation [[26]] but they are weakly connected to the work of this investigation and therefore are not further mentioned here.

\section{1 Intensity Modulation}

An optical sensor with intensity modulation technique is a sensor that uses the light intensity to assess the measurand changes. In the early period of optical fibre sensor development, intensity modulation was the most widely used method for various measurements. This is because the intensity modulation offers the simplest, cheap to construct and most reliable technique [[27]]. However recently, this kind of sensor is seldom used except for simple on/off applications, as intensity modulation method suffers from poor reference signal resulting in measurement inaccuracies [[28]].

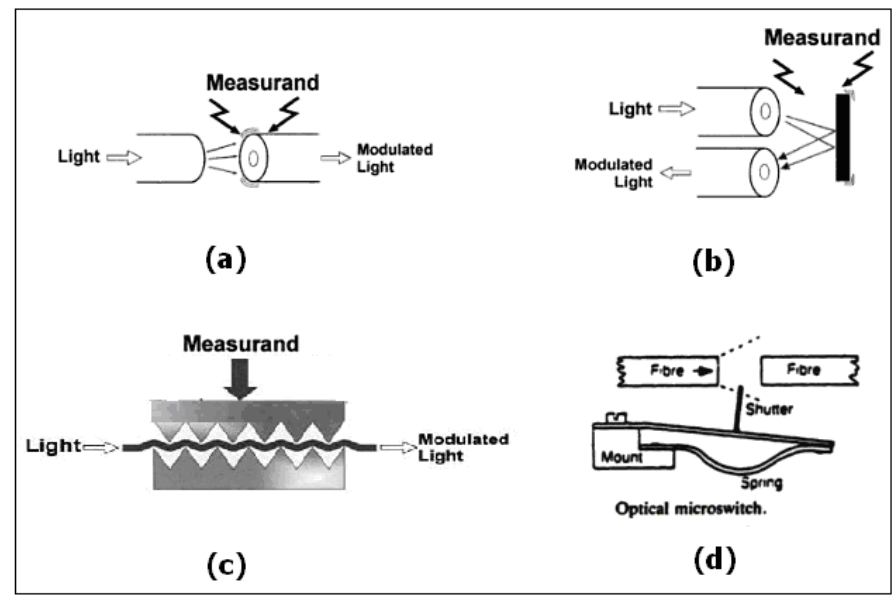

Figure 5: (a) Fibre displacement sensor (b) Reflective sensor

(c) Micro-bend sensor (d) Shutter modulated sensor [[27]].

Loss of intensity due to usage of fibres and connectors is another problem that makes the sensor susceptible to drift. Hence an intensity modulation technique is not suitable for use with less sensitive measurands and for dynamic measurements. Generally, an intensity modulated optical fibre sensor varies the light intensity output with respect to the measurands. There are a number of ways where intensity modulation can be achieved. Techniques include displacement between two fibres, shutter type, reflective and micro-bending. The operating principle of intensity modulation sensors are shown in Figure .

\subsection{Wavelength Modulation}

Wavelength modulation method is an alternative technique used for optical fibre sensing systems. There are currently many wavelength modulation approaches used for different types of sensors. Generally, they comprise a broadband multi-wavelength or tuneable optical source and a sensor element, which acts as an encoder that depends on its perturbation by external parameters. This sensor relies on measured information in terms of specific wavelength of light from the sensor element. The wavelength modulation sensor is well explained in [[29]]-[29] and its basic configuration is shown in Figure 6. 
INTERNATIONAL JOURNAL OF ENGINEERING TECHNOLOGY AND SCIENCES (IJETS) Vol.7 (1) June 2017 DOI: http://dx.doi.org/10.15282/ijets.7.2017.1.7.1069

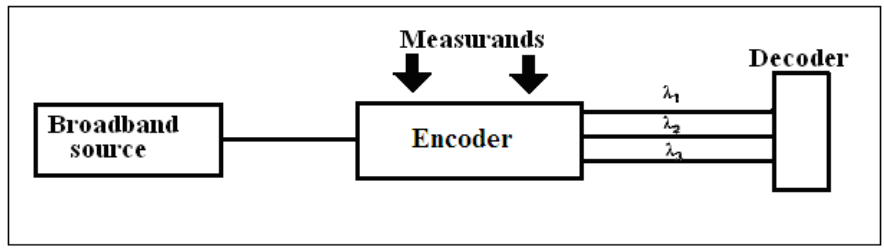

Figure 6: Wavelength modulation sensor.

The wavelength modulated signal can also be realised in Fibre Bragg Grating (FBG) sensors. Each grating can have a particular Bragg wavelength and thus the received wavelength is encoded depending on measurands or external perturbations. Various techniques are used to analyse and decode the signals such as using an electronic filter [[31]], Fourier transform spectrometer [[32]] or Fabry Perot interferometer [[33]]. The FBG provides a good solution whereby all the sensing elements are combined onto a single fibre, resulting in the sensor being more cost effective. This is an example of a true multi point sensor.

\subsection{Time Modulation}

Time modulation sensors exploit the ability of the sensor system to separate each sensing element using a length of the optical fibre, which produces an optical delay in the system. This is illustrated schematically in Figure 7. A pulsed source can be used in order to provide the delay for each sensing part of the system. Each pulse is then in turn reassembled at the receiving end based on synchronised timing. When exposed to measurands, path imbalance occurs in the system and the time difference is captured at the receiving end. The signal at the receiving end can be analysed by an interferometer such as Mach Zehnder or Michelson as reported in the textbookError! Reference source not found. [33]. However in such systems, interferometric noise can happen which can degrade the sensor's performance [[35]]. Interferometric noise is a fluctuation or vibration in the output signal due to an optical interference when the pulses in different channels overlap or when multiple reflections occur between fibre interfaces (connectors, fibre ends etc.) [[36]].

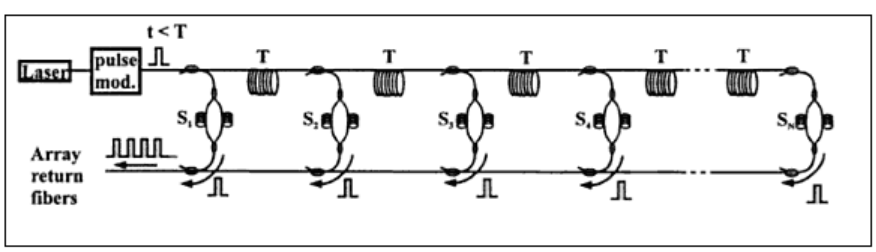

Figure7: Basic time division multiplexed interferometric sensor [34].

\section{SUMMARY}

In this paper an introduction of optical fibres is presented by discussing their usages and progress in telecommunication industries and how it has an indirect impact on the progress of the optical fibre sensing system. Although there are many papers that review on the optical fibre sensor based on different clusters and configurations, this paper only focus on a various types of sensor topology. It also cover a few types of sensor modulation techniques which are intensity modulation, wavelength modulation and time modulation and their working principles were explained in details. The objective of the review is to evaluate their respective suitability for use as a sensor in different environment or it can be used as an alternative method of optical sensing in any intended sector.

\section{ACKNOWLEDGMENT}

The authors are grateful to the staff of Faculty of Engineering Technology for support and helpful discussions as well as constructive suggestions. The authors would also like to acknowledge University Malaysia Pahang for research financial support provided. 
INTERNATIONAL JOURNAL OF ENGINEERING TECHNOLOGY AND SCIENCES (IJETS) Vol.7 (1) June 2017 DOI: http://dx.doi.org/10.15282/ijets.7.2017.1.7.1069

\section{REFERENCES}

[1] William B. Lyons, "A multipoint optical fibre sensors utilising artificial neural network pattern recognition techniques to separate out cross-coupling effects", Phd thesis, Electronic and Computer engineering department, University of Limerick, 2002.

[2] Byoungho Lee, "Review of the present status of optical fiber sensors", Optical Fiber Technology, Vol. 9, Issue 2, April 2003, pp. 57-79.

[3] T.G. Giallorenzi, J.A. Bucaro, A. Dandridge, G.H. Sigel, Jr., J.H. Cole, S.C. Rashleigh, R.G. Priest, "Optical fiber sensor technology", IEEE Journal of Quantum Electronics, Vol. QE-18, 1982, No. 4.

[4] Harmer, A. L., "Principles of optical fibre sensors and instrumentation", Measurement and Control. Vol. 15, Apr. 1982, pp. 143-51.

[5] Alan D. Kersey, “A Review of Recent Developments in Fiber Optic Sensor Technology”, Optical Fiber Technology, Vol. 2, Issue 3, July 1996, pp. 291-317.

[6] K. T. V. Grattan, and T. Sun, "Fiber optic sensor technology: an overview", Sensors and Actuators A: Physical, Vol. 82, Issues 1-3, May 2000, pp. 40-61.

[7] Eric Maurice, GCrard Monnom, D. B. Ostrowsky, Member, ZEEE, and G. W. Baxter, "High Dynamic Range Temperature Point Sensor Using Green Fluorescence Intensity Ratio in Erbium-Doped Silica Fiber", Journal of Lightwave Technology, Vol. 13, No. 7, July 1995, pp. 1349-1353.

[8] Vito Fernicola and Luigi Crovini, "Digital Optical Fiber Point Sensor for High-Temperature Measurement" Journal of Lightwave Technology, Vol. 13, No. 7, July 1995, pp. 1331-1334.

[9] A. Dybko, W. Wróblewski, E. Rozniecka, K.Pozniakb, J. Maciejewski, R. Romaniuk and Z. Brzózka, “Assessment of water quality based on multiparameter fiber optic probe", Sensors and Actuators B: Chemical, Vol. 51, Issues 1-3, August 1998, pp. 208-213.

[10] York Sensors Ltd, website page,

[11] http://www.york-sensors.co.uk/products 800.htm

[12] K. T. V. Grattan, B. T. Meggitt, "Optical Fiber Sensor Technology: Advanced Applications- Bragg Grating and Distributed Sensors", Kluwer Academic Publishers, 2000, Chapter 4, pp. 242.

[13] Ziyi Zhang and Xiaoyi Bao, "Distributed optical fiber vibration sensor based on spectrum analysis of PolarizationOTDR system," Opt. Express 16, 10240-10247 (2008).

[14] M. N. Alahbabil, Y. T. Cho, and T. P. Newson, "Simultaneous temperature and strain measurement with combined spontaneous Raman and Brillouin scattering,” Optics Letters, Vol. 30, no. 11, 2005, pp. 1276-1278.

[15] H. J. Li, K. C. Tan, and Qi Su, "Assessment of Underground Cable Ratings Based on Distributed Temperature Sensing”, IEEE Transaction on Power Delivery, Vol. 21, No. 4, 2006, pp. 1763.

[16] S.H. Eum, K. Kageyama, H. Murayama, K. Uzawa, I. Ohsawa, M. Kanai, S. Kobayashi, H. Igawa and T. Shirai, "Structural health monitoring using fiber optic distributed sensors for vacuum-assisted resin transfer molding", IOP Journal, Smart Material \& Structural 16, 2007, pp. 2627-2635.

[17] Edwin Reynders, Guido De Roeck, Pelin Gundes Bakir, and Claude Sauvage, "Damage Identification on the Tilff Bridge by Vibration Monitoring Using Optical Fiber Strain Sensors", Journal of Engineering, Mech., Vol. 133, Issue 2, February 2007, pp. 185-193.

[18] Banshi Das Gupta, "Fiber Optic Sensors: Principles and Applications", New India Publishing Agency, 2006, Chapter 13, pp. 231.

[19] C. Fitzpatric, E. Lewis, A. Al-Shamma'a and J. Lucas "An Optical Fibre Sensor Based on Cladding Photoluminescence for High Power Microwave Plasma UV Lamps Used in Water Treatment." Optical Review, Vol.8, No.6, Dec 2001, pp. 459-463.

[20] H.Y. Fu, H.L. Liu, H.Y. Tam, P.K.A Wai, C. Lu, "Long-distance and quasi-distributed FBG sensor system using a SOA based ring cavity scheme", Optical Fiber Communication and the National Fiber Optic Engineers Conference, OFC/NFOEC, 2007, pp. 1-3.

[21] G.P. Brady, K. Kalli, D.J. Webb, D.A. Jackson, L. Rekie, and J.L. Archambault, "Simultaneous measurement of strain and temperature using the first and second-order diffraction wavelengths of Bragg gratings", Proc. Inst. Elect. Eng., Optoelectron, Vol. 144, No. 3, 1997, pp. 156-161.

[22] Michael Campbell, Yatao Yang, Peter A. Wallace and A. Sheila Holmes-Smith, "A multipoint quasi-distributed optical fiber pH sensor", The Optical Society of Japan, Vol. 4, No 1, January 1997.

[23] Matjaž Linec and Denis Đonlagić, "Quasi-distributed long-gauge fiber optic sensor system" Optics Express, Vol. 17, Issue 14, 2009, pp. 11515-11529.

[24] Walt Boyes, "Instrumentation reference book", Elsevier Science, 2003, Chapter 12, pp. 171.

[25] Kiyoshi Kurosawa and Satoru Yoshida, "Polarization-maintaining properties of the flint glass fiber for the Faraday sensor element”, Proceeding SPIE, Vol. 2360, 1994, pp. 28-31. 
[26] Chang-Seok Kim, Younggeun Han, Byeong Ha Lee, Won-Taek Han, Un-Chul Paek and Youngjoo Chung,"Induction of the refractive indexnext term change in B-doped optical fibers through relaxation of the mechanical stress",Optics Communications, Vol. 185, Issues 4-6, 15 Nov 2000, pp. 337-342.

[27] A. N. Chester, S. Martellucci, Anna Maria, “Optical fiber sensors” Kluwer Academic Publisher, 1986, pp. 131.

[28] K. T. V. Grattan, B. T. Meggitt, “Optical Fiber Sensor Technology: Devices and Technology”, Chapman \& Hall, Vol. 2, 1998, Chap. 1, pp. 20.

[29] Massood Tabib-Azar, "Integrated optics, microstructures, and sensors", Kluwer Academic Publisher, Section III, Chap. 2, 1995, pp. 248.

[30] K. T. V. Grattan, B. T. Meggitt, “Optical Fiber Sensor Technology: Devices and Technology”, Chapman \& Hall, Vol. 2, 1998, Chap. 4, pp. 147.

[31] A. Irace, G. Breglio and A. Cutolo, "Silicon-based optoelectronic filter based on an electronically active waveguide embedded Bragg grating", Optics Communications, Vol. 221, Issues 4-6, June 2003, pp. 313-316.

[32] M. A. Davis and A. D. Kersey, "Application of a Fiber Fourier Transform Spectrometer to the Detection of Wavelength-Encoded Signals from Bragg Grating Sensors”, Journal og Lightwave Technology, Vol. 13, No. 7, July 1995, pp. 1289-1295.

[33] Y. J. Rao, Z. L. Ran, and C. X. Zhou, "Fiber-optic Fabry-Perot sensors based on a combination of spatial-frequency division multiplexing and wavelength division multiplexing formed by chirped fiber Bragg grating pairs", Applied Optics, Vol. 45, Issue 23, 2006, pp. 5815-5818.

[34] K. T. V. Grattan, B. T. Meggitt, "Optical fiber sensor technology: fundamentals", Kluwer Academic Publisher, 2000, Chap. 1, pp. 10-11.

[35] K. S. Jepsen, H. N. Poulsen, A. T. Clausen, A. Buxens, and K. E. Stubkjaer, "Investigation of cascadability of add-drop multiplexers in OTDM systems", in Proc. ECOC, 1998, pp. 619-620.

[36] Petar K. Pepeljugoski and Kam Y. Lau, "Interferometric Noise Reduction in Fiber-optic Links by Superposition of High Frequency Modulation”, Journal of Lightwave Technology, Vol. 10, No. 7, July 1992, pp.957-963. 